\title{
Parametrized equations for excitons in quantum wires
}

\author{
A. El Haddad, J. Diouri and A. Taqi \\ Faculté des sciences , BP 2121, Tétouan, Morocco, \\ E-mail: a_haddad01@yahoo.fr
}

\begin{abstract}
A set of analytic equations for calculating the binding energies of excitons in T-shaped and squared quantum well wires are established within the effective mass approximation and the two-band model. The resolution is performed in the framework of the variational method. The projections of the relative movement in a lateral plane (2D exciton) and along the free movement direction (1D exciton) are examined as limiting cases. Binding energies and spatial extensions of the exciton as functions of the size of the wire for both the ground and the first excited states are calculated in the case of $\mathrm{GaAs} / \mathrm{GaAlAs}$ heterostructures for T-shaped and squared geometries. The method is applied to calculate the effects on the excitons induced by the application of crossed electric and magnetic fields. Comparison between quantum wells, T-wires and squared wires is given.
\end{abstract}

Keywords: exciton, quantum well wires, heterostructures, binding energy.

Manuscript received 17.03.05; accepted for publication 18.05.05.

\section{Introduction}

Nowadays, the art of growing nanometre-sized quantum well wires (QWRs) with various cross sectional shapes is sufficiently advanced giving rise to new optical performances not achievable in 2D systems (for reviews, see [1-3] and references therein). The literature abounds on studying optical and electrical properties of a wide variety of such structures involving II-VI (CdMnS, CdMnSe) and III-V (GaAlAs, GaInAs / InP) compounds [3-5]. Over the past ten years, self-organized growth and cleaved edge overgrowth techniques have been developed to fabricate high-quality samples (V-groove and T-shaped wires) with enhanced interface uniformity [4-5]. It has been proved that for such structures, the confinement of the carriers in the edge region (at $10 \mathrm{~nm}$ scale regime) acts as a quasi-1D confinement enhancing then the binding energy of the excitons as well as the fundamental absorption intensity. A large number of theoretical and experimental works have been devoted to study excitonic effects in cylindrical, $\mathrm{V}$-groove and $\mathrm{T}$ shaped wires [6-23]. In general, binding energies of excitons $E_{b}$ in such systems were calculated using various theoretical approaches and numerical techniques. In [13], a first calculation of $E_{b}$ for GaAs rectangular quantum wire (RQWR) surrounded by an infinite confining potential and including phonon effects was performed with the use of variational solution to the effective mass approximation. Excitons in cylindrical quantum wires (CQWR) with infinite [24] and finite [11] barrier were studied and binding energies were calculated analytically for the first case and variationally for the second. In [25], a numerical solution of the Bloch equations describing the multisubband " $1 \mathrm{D}$ " system was used to analyze optical properties of T- and V-like wires based on GaAlAs / GaAs structures. As a whole, it has been found that $E_{b}$, as a function of the cross-section dimensions, follows trends similar to those habitually observed for 2D quantum wells but with top values larger than the ideal 2D limit. In general, the theoretical treatment of excitons in QWRs requires first to solve the two-dimensional Schrödinger equation for the confined states of the wire. To do this, except for cylindrical wires, which are completely solved analytically [24, 11], various numerical approaches were used to solve equations governing the eigenstates of electrons and holes mainly for realistic structures, i.e., T- and V-wires. Today, the tendency in investigating quantum wires is in general limited to the so-called realistic systems, i.e., Tand V-wires after some extensive studies of CQWRs. However, RQWRs are susceptible to give interesting features for both basic physics and applications. Effectively, in RQWRs, the confinement is expected to be stronger and, as a consequence, the excitons more enclosed: further localization and higher binding energies. This would yield more intensive and more squeezed excitonic spectra susceptible with new interesting applications. In addition, it was mentioned that these systems allow, when growing, enough precision in controlling shape and size even in the nanoscale region [26]. Furthermore, the theoretical 
treatment of such structures enables, in a relatively simpler way, the derivation of quasi-analytical equations for excitonic states provided that electron and hole states are known. Actually, whereas the exciton properties for $\mathrm{T}$ - and V-like geometries are widely studied and compared each to other revealing no significant difference [25], it is still unclear whether the shape of cross-section influences the exciton properties when they are compared to the square-like geometry. Examination of the available data makes the response to this question difficult because of the wide variety of the physical parameters used, for the same material, in calculating and interpreting these properties.

The aim of the present paper is to elucidate some aspects of these questions by focusing on the exciton properties in squared cross-section quantum wires (SQWRs). The corpus of the paper is a generalization of the method we have developed for quantum wells (QWs) with rectangular and parabolic confinements [27]. In contrast with QWs, the main difficulty for the analytic study of RQWRs arises from the coupling between $x$ and $y$ movements introduced by the discontinuity of the confining potential along $(x-y)$-directions making the factorization (with respect to $x$ and $y$ coordinates) of the single particle function not relevant. A few methods have been suggested to solve electron and hole states for RQWR with finite potential barrier [9 and references therein]. Recently, M. Tsetseri et al. [28] have used the finite difference method to calculate the ground state of $\mathrm{V}$ and rectangular quantum wires. They found that the energy is strongly affected by the shape of the wire and showed that $\mathrm{V}$ - and $\mathrm{T}$-wires may be approximated by rectangular wires with suitable sides ratio. In the particular case of SQWRs which approximate the symmetric T-wires, factorized wavefunctions with required boundary conditions at the interfaces may be used as solutions for the ground state.

In order to find practical and relatively simplified equations for excitons in QWRs, we develop a method where advanced analytical steps are carried out. The method is performed in the framework of the envelope function formalism based on the effective mass approximation and two-band model including valence band anisotropy. The solution is found on the basis of the variational principle. One power of the method is that it can be applied to any potential profile, provided that the solution of the one particle problem is known. We have also extended the method to study the effects of applied electric and magnetic fields on excitons. The paper is organized as follows: in the following section we formulate the assumptions and the equations of the model. The expressions for the spatial extension and the binding energy of the exciton are then established. The $2 \mathrm{D}$ and $1 \mathrm{D}$ limiting cases, as projections of the relative movement in the lateral plane and along the freemovement direction, respectively, are deduced as a test of the validity of the model. Applications of the model to T-shaped and squared quantum wires with and without the presence of electric and magnetic fields are given and discussed in Section 3.

\section{The model}

\subsection{Basic equations}

Let us consider a QWR, of type I extending infinitely along the $z$-direction with $L_{x}$ and $L_{y}$ transversal dimensions. The confining potential is of arbitrary shape, say $V_{e}\left(x_{e}, y_{e}\right)$ for electrons and $V_{h}\left(x_{h}, y_{h}\right)$ for holes. In the hypothesis of uncoupled excitons and within the envelope function approximation, the Hamiltonian operator for the bound states of one exciton $X$ reads, in the effective mass approximation:

$H=H_{e}+H_{h}+H_{X}$

where

$H_{i}=-\frac{\partial}{\partial x_{i}}\left(\frac{1}{m_{i}} \frac{\partial}{\partial x_{i}}\right)-\frac{\partial}{\partial y_{i}}\left(\frac{1}{m_{i}} \frac{\partial}{\partial y_{i}}\right)+V_{i}\left(x_{i}, y_{i}\right)$

and

$H_{X}=-\frac{1}{\sigma} \frac{\partial^{2}}{\partial Z^{2}}-\frac{2}{r}$.

Here $H_{i}$ describes the $(x, y)$-movement of the carrier $i$ $(i=e, h)$ in the presence of the confining potential $V_{i}\left(x_{i}, y_{i}\right)$ with the ground state energy $E_{i}$. In equation (3), $Z$ is the relative position between the electron and the hole along $z$-axis: $Z=z_{e}-z_{h}, r$ is their relative distance, $\mu_{z}$ is the reduced mass along the $z$-axis and $\sigma=\frac{\mu_{z}}{\mu^{*}}$ where $\mu^{*}$ is the reduced electron-hole mass ratio defined by: $\frac{1}{\mu^{*}}=\frac{1}{m_{e}^{*}}+\frac{1}{m_{h}^{*}}, m_{e, h}^{*}$ being the effective mass of the electron $(e)$ or the hole $(h)$. In this formulation, effective atomic units are used, i.e., $a_{B}=\frac{\hbar^{2} \varepsilon}{\mu^{*} e^{2}}$ for distance, $R=\frac{\mu^{*} e^{4}}{2 \hbar^{2} \varepsilon^{2}}$ for energy and $\mu^{*}$ for masses. $\varepsilon$ represents the dielectric constant which is set to be equal inside and outside the well, neglecting then the dielectric mismatch effect which is minor in comparison to the confinement effect mainly in the strong confinement regime [25]. The exciton problem is then solved variationally: the energies $E$ and the wavefunctions $\Psi\left(\vec{r}_{e}, \vec{r}_{h}\right)$ of the exciton are determined by minimizing the expected values of $H$ with respect to a suitable set of variational parameters and the binding energies $E_{b}$ are deduced from the relation:

$E_{b}=E_{e}+E_{h}-E$.

Owing to the hydrogen-like coupling between the electron and the hole, the most appropriate trial wavefunction may be set as follows:

$\Psi=\Psi_{e}\left(x_{e}, y_{e}\right) \Psi_{h}\left(x_{h}, y_{h}\right) \Phi$,

where the coupling factor $\Phi$ is chosen conveniently to describe the exciton state, and $\psi_{e}$ and $\psi_{h}$ are obtained 
by solving the Hamiltonian (2) for the electron and the hole respectively. Thus, for the ground state $X_{l s}$, we take:

$\Phi_{1 s}=\exp \left(-\frac{r^{\prime}}{\lambda}\right)$

and for the first excited state

$\Phi_{2 s}=\left(1-\frac{r^{\prime}}{\lambda}\right) \exp \left(-\frac{r^{\prime}}{\lambda}\right)$

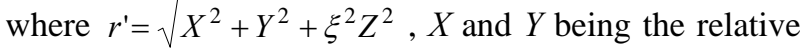
positions in the $(x-y)$ plane: $X=x_{e}-x_{h}$ and $Y=y_{e}-y_{h} . \lambda$ and $\xi$ are the variational parameters.

\subsection{General solutions}

In order to preserve the generality of the problem, we will derive the parameterized formula in terms of the coupling factor $\Phi$ and its derivatives. Substituting (5) into the Schrödinger equation described by the Hamiltonian (1) and taking into account of (4), we obtain:

$$
\begin{aligned}
& E_{b}\langle\Psi \mid \Psi\rangle=-\int\left[\frac{1}{m_{e}}\left(\left(\frac{\partial \Phi}{\partial x_{e}}\right)^{2}+\left(\frac{\partial \Phi}{\partial y_{e}}\right)^{2}\right)+\right. \\
& +\frac{1}{m_{h}}\left(\left(\frac{\partial \Phi}{\partial x_{h}}\right)^{2}+\left(\frac{\partial \Phi}{\partial y_{h}}\right)^{2}\right)+\frac{1}{\sigma}\left(\frac{\partial \Phi}{\partial Z}\right)^{2}- \\
& \left.-\frac{2}{r} \Phi^{2}\right] \times \Psi_{e}^{2} \Psi_{h}^{2} d \Omega
\end{aligned}
$$

with $d \Omega=d x_{e} d x_{h} d y_{e} d y_{h} d Z$.

Since the effective mass mismatch effect in calculating the binding energy of the exciton is very small as mentioned in the previous work [27], the integration operations in equation (8) may be performed over all the structure by making constant the effective mass, equalled to its value in the well. The calculation reduces then to some analytic manipulations involving the coupling factor $\Phi$. For details of the procedure see reference [27]. In the present case, the parameterized binding energy writes:

$E_{b}=\frac{2 \mathfrak{I}(K)-\mathfrak{I}(J)}{\mathfrak{I}(F)}$,

where the operator $\mathfrak{I}$ is defined by

$$
\Im(A)=\int_{0}^{\infty} \int_{0}^{\infty} P(X, Y) A(X, Y) d X d Y .
$$

$P(X, Y)$ being the probability of finding the electron and the hole separated by $X$ and $Y$ which writes:

$$
\begin{aligned}
& P(X, Y)=\int \psi_{e}^{2}(x+X, y+Y) \psi_{h}^{2}(x, y) d x d y+ \\
& +\int \psi_{h}^{2}(x+X, y+Y) \psi_{e}^{2}(x, y) d x d y .
\end{aligned}
$$

In these equations, the entities $F, J, K$, and $G$ are given by:

$$
\begin{aligned}
& F(X, Y)=\int \Phi^{2} d Z ; \\
& J(X, Y)=\int\left(\left(\frac{\partial \Phi}{\partial x_{e}}\right)^{2}+\left(\frac{\partial \Phi}{\partial y_{e}}\right)^{2}+\frac{1}{\sigma}\left(\frac{\partial \Phi}{\partial Z}\right)^{2}\right) d Z \\
& K(X, Y)=\int \frac{\Phi^{2}}{r} d Z ; G(X, Y)=\int \Phi^{2} Z^{2} d Z .
\end{aligned}
$$

In the same way, the spatial extensions of the exciton along the axes express simply as follows:

$$
\begin{aligned}
& X_{e x}^{2}=\left\langle X^{2}\right\rangle_{\psi}=\frac{\mathfrak{I}\left(X^{2} F\right)}{\mathfrak{I}(F)} ; Y_{e x}^{2}=\left\langle Y^{2}\right\rangle_{\psi}=\frac{\mathfrak{I}\left(Y^{2} F\right)}{\mathfrak{I}(F)} ; \\
& Z_{e x}^{2}=\left\langle Z^{2}\right\rangle_{\psi}=\frac{\mathfrak{I}(G)}{\mathfrak{I}(F)} .
\end{aligned}
$$

It results from these equations that the crucial step for solving the exciton states is to know the representative function $P(X, Y)$, denoted hereafter by the $P$-function, which is in turn function of the one-particle wavefunctions. Thus, the proceeding in what follows consists in determining first the one-particle states and then the $P$-function from which the parameterized equations of the binding energies and extensions of the exciton are deduced via equations (9) and (13). Before turning to the applications of the method, let's note that the parameterized expression of the energy, equation (9), is in fact the sum of the rinetic part of the energy, i.e.,

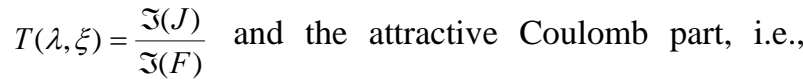
$U(\lambda, \xi)=-\frac{2 \mathfrak{I}(K)}{\mathfrak{I}(F)}$ which makes it easy, after minimization the total energy functional, to deduce the kinetic and the electric potential energies in terms of the entities $\mathfrak{I}(F), \mathfrak{I}(J)$ and $\mathfrak{I}(K)$.

To make easy the use of our results for desirable cases, we have preserved the generality by using dimensionless units, i.e., $R=a_{B}=1$ where $R$ and $a_{B}$ are the Rydberg energy and the Bohr radius, respectively, of bulk exciton for well material.

\subsection{Limiting cases}

Before giving the variations of the exciton properties for various shapes and sizes of the wire, it is interesting to examine how the equations established above transform within the limiting cases of three, two and one dimensional structures. Indeed, the asymptotic behaviour of the exciton with these limits is independent of the cross-sectional shape, and it may be used as a test of the validity of the model. The former case corresponds to the situation where the exciton is quasi-free, as in the bulk material; for the second case both electron and hole are assumed to be confined in a same plane, while in the latter case the confinement is supposed to be exactly of the one-dimensional one. 
a) 3D-exciton. Approaching the bulk-well material limit ( $L_{x} \rightarrow \infty$ and $L_{y} \rightarrow \infty$ ), the confinement functions $\psi_{e}$ and $\psi_{h}$ become smooth, i.e. $\partial_{X, Y} \approx 0$ and taking into account the spherical symmetry $(\xi \approx 1)$, we obtain:

$\frac{\mathfrak{I}(J)}{\mathfrak{I}(F)}=\frac{2 \sigma+1}{3 \sigma} \frac{1}{\lambda^{2}} ; \frac{\mathfrak{I}(K)}{\mathfrak{I}(F)}=\frac{1}{\lambda} ; \frac{\mathfrak{I}(G)}{\mathfrak{I}(F)}=\lambda^{2}$.

Substituting these expressions into equation (9) and making $\sigma=1$, as required for isotropic masses, we obtain: $E_{b, 1 s}^{3 D}(\lambda)=-\frac{1}{\lambda^{2}}+\frac{2}{\lambda}$. Maximizing then with respect to the variational parameter $\lambda$ yields: $E_{b, 1 s}^{3 D}=1$ with corresponding the Bohr radius $a_{1 s}^{3 D}=\lambda_{1 s}^{3 D}=1$ and $2 T=-U=2$ as expected. The corresponding extensions are: $Z_{1 s}^{3 D}=Y_{1 s}^{3 D}=X_{1 s}^{3 D}=a_{1 s}^{3 D}=1$. Let's note that this case corresponds also to the limiting situation of infinitely narrow wire $\left(L_{x} \rightarrow 0, L_{y} \rightarrow 0\right)$ where the exciton takes the bulk-barrier character: the carrier wave function spreads and penetrates into the barriers making the binding energy decrease towards the value appropriate to the bulk barrier material (which is supposed here to be equal to that of the bulk well material because of the approximation made of equalling masses and dielectric constants)

Similarly, for the $2 s$-exciton, we obtain, with 3Dlimiting $\quad$ case: $\quad \frac{\mathfrak{I}(J)}{\mathfrak{I}(F)}=\frac{2 \sigma+1}{3 \sigma} \frac{1}{\lambda^{2}} ; \quad \frac{\mathfrak{I}(K)}{\mathfrak{I}(F)}=\frac{1}{2 \lambda}$; $\frac{\mathfrak{I}(G)}{\mathfrak{I}(F)}=\frac{7}{2} \lambda^{2}$, which yields, after maximizing: $E_{b, 2 s}^{3 D}=\frac{1}{4} \quad\left(\frac{3 \sigma}{1+2 \sigma}\right)=\frac{1}{4} \quad ;$ with $a_{2 s}^{3 D}=2\left(\frac{1+2 \sigma}{3 \sigma}\right)=2$ and $2 T=-U=\frac{1}{2}, Z_{2 s}^{3 D}=X_{2 s}^{3 D}=Y_{2 s}^{3 D}=\sqrt{\frac{7}{2}} \lambda_{2 s}^{3 D}=\sqrt{14}$.

b) 2D-exciton. This case corresponds formally to the state for which the binding energy reaches its top limit value: both the electron and hole are assumed to be confined in the same plane $\left(X=0, L_{y} \rightarrow \infty\right)$. It may be considered as the projection of the relative movement of the exciton in the $(y, z)$-plane. The coupling factor $\Phi$ is thus given by: $\Phi_{1 s}=\exp \left(-\frac{\rho}{\lambda}\right)$ where $\rho$ is the polar radius in the $(\mathrm{y}, \mathrm{z})$-plane. Then, we obtain, for isotropic masses $\quad(\sigma=1): \quad \frac{\mathfrak{I}(J)}{\mathfrak{I}(F)}=\frac{1}{\lambda^{2}} ; \quad \frac{\mathfrak{I}(K)}{\mathfrak{I}(F)}=\frac{2}{\lambda} \quad$ and $E_{b, 1 s}^{2 D}(\lambda)=-\frac{1}{\lambda^{2}}+\frac{4}{\lambda}$. Maximizing then with respect to $\lambda$ gives: $E_{b, 1 s}^{2 D}=4, \quad a_{1 s}^{2 D}=\frac{1}{2}, \quad$ as expected, with $2 T=-U=8 \quad$ and: $\quad Z_{1 s}^{2 D}=Y_{1 s}^{2 D}=\frac{\sqrt{3}}{2} a_{1 s}^{2 D}=\frac{\sqrt{3}}{4} \quad$ and consequently: $r_{1 s}^{2 D}=\sqrt{\frac{3}{8}} a_{B}$, in agreement with the expression given by G.W. Bryant et al. [29].
For the 2s exciton, within the 2D-limiting case, similar calculation gives: $\frac{\mathfrak{I}(J)}{\mathfrak{I}(F)}=\frac{1}{\lambda^{2}} ; \quad \frac{\mathfrak{I}(K)}{\mathfrak{I}(F)}=\frac{2}{3 \lambda}, \quad E_{b, 2 s}^{2 D}=$ $=\frac{4}{3 \lambda}-\frac{1}{\lambda^{2}}$ and then $E_{b, 2 s}^{2 D}=\frac{4}{9} \quad$ with $a_{2 s}^{2 D}=\frac{3}{2}$ and $2 T=-U=\frac{8}{9} ; \quad Z_{2 s}^{2 D}=Y_{2 s}^{2 D}=\frac{a_{2 s}^{2 D}}{2}=\frac{3}{4}$. Note that the coupling factor in this case is given by the first excited state of the 2D-hydrogen atom, i.e., $\phi_{2 s}^{2 D}=\left(1-\frac{2}{\rho}\right) e^{-\frac{\rho}{\lambda}}$.

c) 1D-exciton. This second formal case corresponds to the theoretical complete confinement along the two directions; the two particles being free only along the $z$ axis. It is in fact the projection of the relative movement along this axis. Thus, it can be assimilated to the problem of a one-dimensional hydrogen atom. In this case, the admissible coupling factor may be approached by [30]: $\quad \phi_{1 s}^{1 D}=|x| \exp (-|x| / \lambda)$ for the $1 s$ exciton and $\phi_{2 s}^{1 D}=\left(|x|-x^{2} / \lambda\right) \exp (-|x| / \lambda)$ for the $2 s$ exciton. Which gives, for the former case $E_{b, 1 s}^{1 D}=R ; \lambda_{1 s}^{1 D}=a_{B} ; X_{1 s}^{1 D}=$ $=\sqrt{3} a_{B}$ and for the second, $E_{b, 2 s}^{1 D}=\frac{R}{4} E ; \lambda_{2 s}^{1 D}=2 a_{B}$.

As it can be seen, this result, obtained in elementary way, agrees with that obtained by $\mathrm{M}$. Combescot et al. [31] in the framework of a complete theoretical study.

\section{Applications}

We have applied the method described above to study excitons in realistic T-wires and SQWRs based on $\mathrm{Ga}_{1-\mathrm{x}} \mathrm{Al}_{\mathrm{x}} \mathrm{As} / \mathrm{GaAs}$ structures. As it arises from equations (9)-(13), the key of the problem consists in finding the characteristic $P$-function which depends exclusively on electron and hole states of the structure. For the hole's ground state, we have considered only the heavy hole neglecting then the valence band mixing [32, 33]. The correspondent mass components were calculated in terms of the Luttinger band parameters [7], i.e. $m_{h h}^{x y}=\frac{m_{0}}{\gamma_{1}+\gamma_{2}} \quad$ and $\quad m_{h h}^{z}=\frac{m_{0}}{\gamma_{1}-2 \gamma_{2}}$. The physical parameters used for numerical applications are listed in Table 1.

Table 1. Physical parameters used for the calculations of binding energies and spatial extensions of the excitons.

\begin{tabular}{|c|c|c|c|}
\hline Symbol & Parameter & Unit & $\mathrm{Ga}_{1-\mathrm{x}} \mathrm{Al}_{\mathrm{X}} \mathrm{As}$ \\
\hline $\begin{array}{c}E_{g} \\
\Delta E_{c} / \Delta E_{v} \\
m_{e}\end{array}$ & $\begin{array}{c}\text { energy gap } \\
\text { band offsets } \\
\text { electron mass }\end{array}$ & $\begin{array}{l}\mathrm{eV} \\
m_{0}\end{array}$ & $\begin{array}{c}1.519+1.138 x+0.47 x^{3} \\
67 / 33 \\
1 /(14.9-8.2 x)\end{array}$ \\
\hline $\left.\begin{array}{l}\gamma_{1} \\
\gamma_{2}\end{array}\right\}$ & $\begin{array}{l}\text { Luttinger } \\
\text { parameters }\end{array}$ & & $\begin{array}{c}7.1-3.34 x \\
2.02-1.12 x\end{array}$ \\
\hline
\end{tabular}




\subsection{SQWRS}

In this case, the potential (for both electron and hole) is supposed to be zero in the well and constant in the barrier say:

$V_{i}\left(x_{i}, y_{i}\right)=\left\{\begin{array}{l}0 \quad \text { for }\left|x_{i}\right|<L / 2 ;\left|y_{i}\right|<L / 2 \\ V_{0, i} \text { otherwise }\end{array}\right.$

where $L$ is the side width of the wire. To show the suppleness of the method, we first consider the case of infinite confining potential $\left(V_{0, i}=\infty\right)$. The one particle ground state is then fully described by the wave function:

$\psi_{i}\left(x_{i}, y_{i}\right)=\cos \left(\frac{\pi}{L} x_{i}\right) \cos \left(\frac{\pi}{L} y_{i}\right)$

It follows that the $P$-function takes the form: $P(X, Y)=P_{x}(X) P_{y}(Y)$ with:

$P_{x}(X)=(L-X)\left(\frac{1}{4}+\frac{1}{2} \cos ^{2}\left(\frac{\pi X}{L}\right)\right)+$

$+\frac{3 L}{4 \pi} \cos \left(\frac{\pi X}{L}\right) \sin \left(\frac{\pi X}{L}\right)$

and similar expression for $P_{y}(Y)$, what reduces considerably the numerical calculation steps.

For a finite barrier height $V_{0, i}$, variable-separable functions

$\psi_{i}\left(x_{i}, y_{i}\right)=f_{i}\left(x_{i}\right) g_{i}\left(y_{i}\right)$

were used as a solution of the one particle problem with

$f_{i}\left(x_{i}\right)= \begin{cases}\cos \left(k_{x, i} x_{i}\right) & \text { for }\left|x_{i}\right|<L / 2 \\ \cos \left(k_{x, i} L / 2\right) \exp \left(-k_{x, i}\left(\left|x_{i}\right|-L / 2\right)\right) & \text { for }\left|x_{i}\right|<L / 2\end{cases}$

and similar equation for $g_{i}\left(y_{i}\right)$. The parameters $K_{x, i}=K_{y, i}=K_{i}=\sqrt{m_{b, i}}\left(V_{0, i}-E_{i}\right) / 2$ and $k_{x, i}=k_{y, i}=$ $=k_{i}=\sqrt{m_{w, i} E_{i} / 2}$ were determined by requiring continuity of $\psi$ and its derivatives $\psi^{\prime} / m$ at interfaces which leads to the energy equation

$\frac{k_{i}}{m_{w, i}} \operatorname{tg}\left(k_{i} \frac{L}{2}\right)=\frac{K_{i}}{m_{b, i}}$.

For $L=5.5 \mathrm{~nm}$, we find $E_{e}=108 \mathrm{meV}$ for the fundamental state. This value is in very good agreement with that obtained by M. Tsetseri et al. [28] where the finite difference method is used. The analytical expressions for electron and hole states given by equation (19) were thus used for determining the $P$-function of the exciton. In this case too, that function separates as a product of two symmetric components $P_{x}(X)$ and $P_{y}(Y)$ but not takes a simple analytical form.
So it was calculated numerically. In Fig. 1, we report the binding energy (a), the extensions $Z_{e x}$ (b) and $\rho_{e x}$ (c) of the heavy hole exciton $\left(X_{1 \mathrm{~s}}\right)$ as a function of the width of the wire for different values of $x$-Al concentration. As it is seen, for each value of $x$, the binding energy increases with decreasing $L$, reaches a maximum value and decreases monotonically. All the curves extrapolate to the exact value of the bulk material at vanishing and infinite $L$ (barrier material for $L \rightarrow 0$ and well material for $L \rightarrow \infty$ ). The peak in energy occurs for $L$ ranging between 0.2 and 0.3 . It corresponds to the case for which the probability of finding the electron and the hole outside the well is negligible. Concerning the extensions, Fig. 1b,c highlights clearly the effect of the confinement: the exciton is compressed in both transversal and longitudinal directions, and compresses as well as the barrier rises. Here also, $Z_{e x}$ and $\rho_{e x}$ approach the bulk material values for large well widths, decrease with lowering $L$ with a pronounced minimum located at around the value maximizing the binding

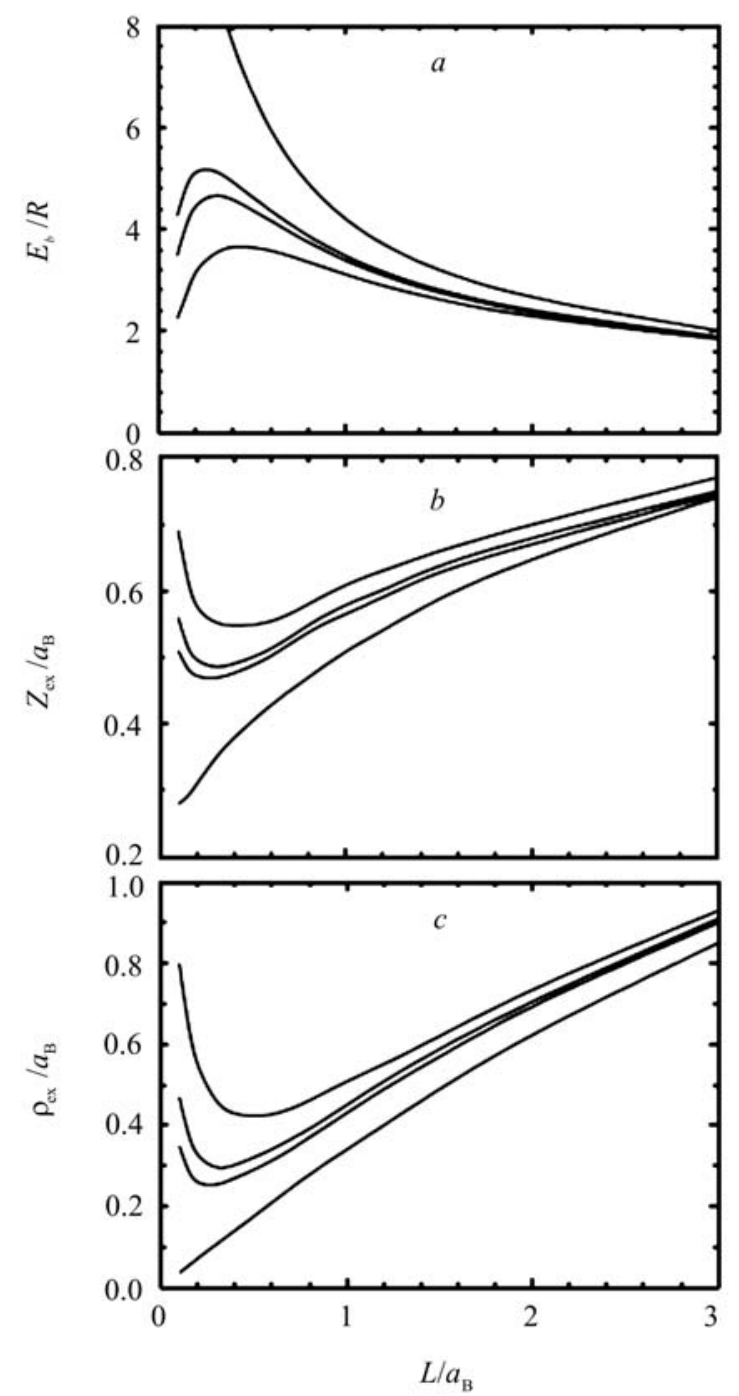

Fig. 1. $L$-dependence of the binding energy (a), $z$-extension (b) and the in-plane extension (c) of the $X_{1 s}$ exciton for the SQWR based on $\mathrm{Al}_{\mathrm{x}} \mathrm{Ga}_{1-\mathrm{x}} \mathrm{As} / \mathrm{GaAs} / \mathrm{Al}_{\mathrm{x}} \mathrm{Ga}_{1-\mathrm{x}} \mathrm{As}$ structure for $x=0.15$, $0.30,0.40$ and infinite barrier (from down to up for the energy, inversely for the extensions). 
energy, and increase again approaching the bulk shape for infinitely narrow QWW. This behaviour is similar to that obtained for the exciton in a rectangular quantum well reported recently in reference [27]. For the infinite barrier, both $Z_{e x}$ and $\rho_{e x}$ decrease monotonically with decreasing $L$. Characteristics of the excited state of the exciton $\mathrm{X}_{2 \mathrm{~s}}$ for the same structure are shown in Fig. 2 for a finite barrier $(x=0.4)$. They present the same behaviour than the $1 s$ exciton with a maximum of 4.60 reached at about $L=0.3$.

We have also examined the theoretical case where the confining potential is parabolic along the $x$-direction and rectangular along the $y$-direction, say

$$
V_{i}\left(x_{i}, y_{i}\right)= \begin{cases}4 V_{0, i} \frac{x_{i}^{2}}{L_{x}^{2}} \text { for }\left|x_{i}\right|<L_{x} / 2 \text { and }\left|y_{i}\right|<L_{y} / 2 \\ V_{0, i} & \text { for }\left|x_{i}\right|>L_{x} / 2 \text { and }\left|y_{i}\right|<L_{y} / 2 \\ \infty & \text { for }\left|y_{i}\right|<L_{y} / 2\end{cases}
$$

Then, the one particle wavefunction can be taken as:

$$
\begin{aligned}
& \psi_{i}\left(x_{i}, y_{i}\right)=\exp \left(-\frac{1}{2} \alpha_{i} x_{i}^{2}\right) \cos \left(\frac{\pi}{L_{y}} y_{i}\right) \\
& \text { with } \alpha_{i}=2 \frac{\sqrt{V_{0, i} m_{i}}}{L_{x}} .
\end{aligned}
$$
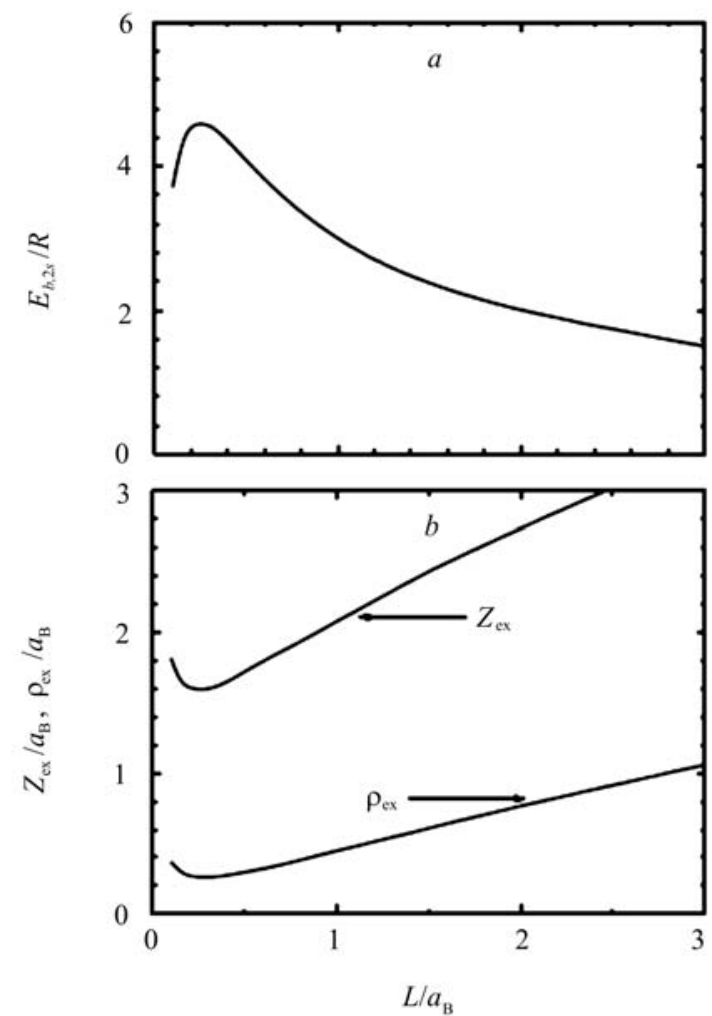

Fig. 2. $L$-dependence of the binding energy (a), z-extension and the in-plane extension (b) of the $X_{2 s}$ exciton for the SQWR based on $\mathrm{Al}_{0.4} \mathrm{Ga}_{0.6} \mathrm{As} / \mathrm{GaAs} / \mathrm{Al}_{0.4} \mathrm{Ga}_{0.6}$ As structure.
It is easy to show that in these conditions the function $P_{x}(X)$ may be calculated analytically and takes the following form:

$P_{x}(X)=\frac{2 \sqrt{\pi}}{\sqrt{\alpha_{e}+\alpha_{h}}} \exp \left(-\frac{\alpha_{e} \alpha_{h}}{\alpha_{e}+\alpha_{h}} X^{2}\right)$

whereas $P_{y}(Y)$ is given by equation (17) with the relevant coordinates.

In Fig. 3a we report the $1 s$ exciton binding energy in GaAs- $\mathrm{Al}_{0.4} \mathrm{Ga}_{0.6}$ As quantum wire, within the latter confining potential configuration, as a function of $L_{x}$-wire width for different values of $L_{y}$. Fig. 3b, c shows the $z$-extension and the in-plane extension of the exciton for the same structure. We note that this confining potential geometry enhances the binding energy and enforces the localization of the exciton comparable to the previous studied geometry.

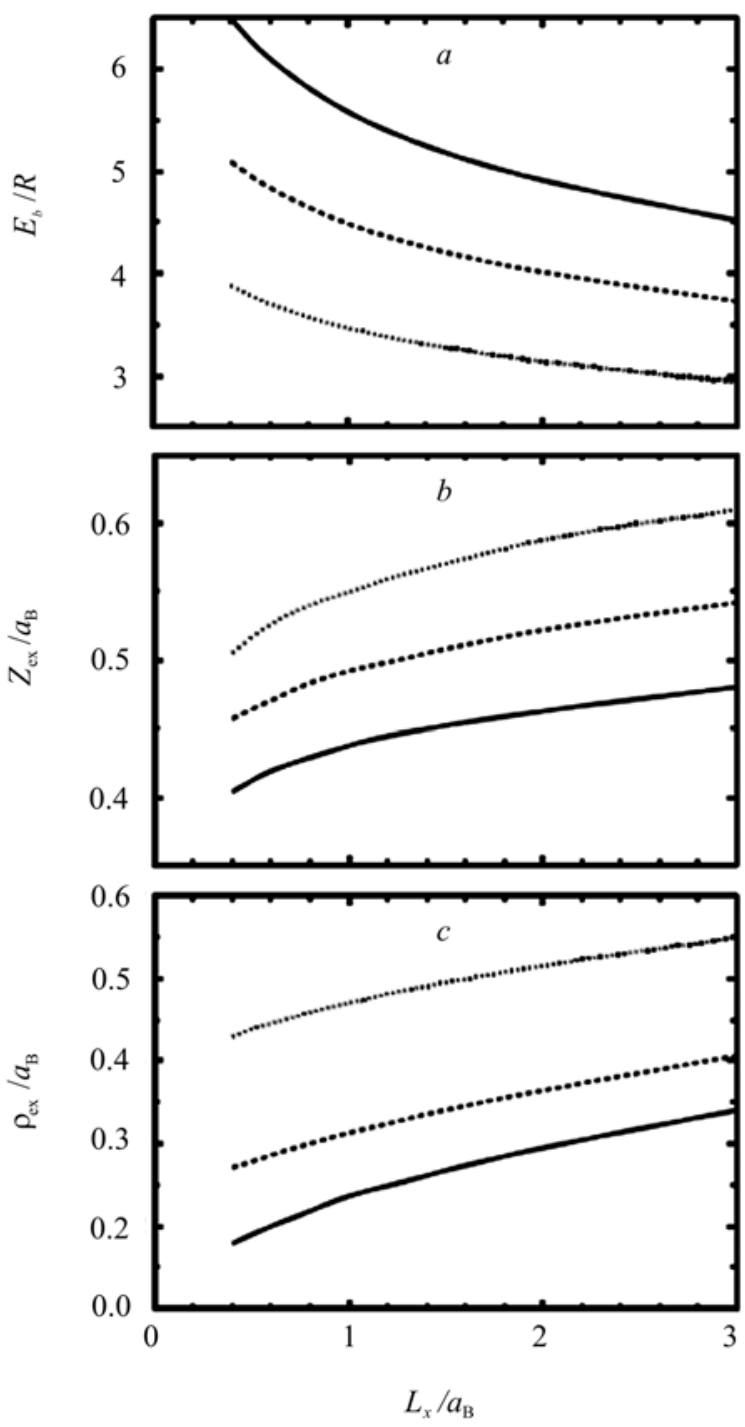

Fig. 3. $L_{x}$-dependence of the binding energy (a), z-extension (b) and the in-plane extension (c) for $L_{y}=0.5$ (solid), $L_{y}=1$ (dashed) and $L_{y}=2$ (dotted) of the $X_{l s}$ exciton in rectangular-parabolic confining potential for $\mathrm{Al}_{0.4} \mathrm{Ga}_{0.6} \mathrm{As} / \mathrm{GaAs} / \mathrm{Al}_{0.4} \mathrm{Ga}_{0.6} \mathrm{As}$ squared cross-section wire. 
Table 2. Values of the binding energy $E_{b}$, the Bohr radius $a$, and the extensions $X, Y, Z$ of $1 s$ (2s) excitons for the limiting cases of 3D, 2D and 1D structures. The values are in the Rydberg energy and the Bohr radius of the bulk GaAs material (see Table 1).

\begin{tabular}{|c|c|c|c|c|c|}
\hline & $E_{b}^{1 s}\left(E_{b}^{2 s}\right)$ & $a^{1 s}\left(a^{2 s}\right)$ & $X^{1 s}\left(X^{2 s}\right)$ & $Y^{1 s}\left(Y^{2 s}\right)$ & $Z^{1 s}\left(Z^{2 s}\right)$ \\
\hline 3D & $1\left(\frac{1}{4}\right)$ & $1(2)$ & $1(\sqrt{14})$ & $1(\sqrt{14})$ & $1(\sqrt{14})$ \\
\hline 2D & $4\left(\frac{4}{9}\right)$ & $\frac{1}{2}\left(\frac{3}{2}\right)$ & - & $\frac{\sqrt{3}}{4}\left(\frac{\sqrt{3}}{2}\right)$ & $\frac{\sqrt{3}}{4}\left(\frac{\sqrt{3}}{2}\right)$ \\
\hline 1D & $1\left(\frac{1}{4}\right)$ & $1(2)$ & - & - & $\sqrt{3}(\sqrt{42})$ \\
\hline
\end{tabular}

\subsection{T-Wires}

For the T-shaped wire, it is not easy to get the one particle function in a simple and analytic form likely to facilitate the calculation of the $P$-function. Nevertheless, we have found that a judicious transformation of the coordinates reduces the problem to that of two separate quantum wells. Successful and precise results were obtained for the first three electronic levels of T-wires with various dimensions of the wire. Details of these calculations will be published soon. The $P$-function, and then binding energies $E_{b}$ of excitons for T-wires, were thus deduced easily in the same way as for the case of SQWR. We initially tested the method by confronting our results with those reported in the literature for the case of AlAs/GaAs T-wires. Referring to the structure studied in [34] for which the same physical parameters were ued, we have calculated $E_{b}$ of $\mathrm{X}_{1 \mathrm{~s}}$ excitons for $L=5.3 \mathrm{~nm}$, as stem width, and $d$ ranging between 4.5 and $6 \mathrm{~nm}$, as arm width. Our calculated values were ranged between 22.41 and $19.61 \mathrm{meV}$, slightly lower than those roughly estimated in that reference according to photoluminescence spectra analysis. In turn, our values are clearly higher than those obtained by G. Goldoni et al. [25] where the calculation is based on multisubband semiconductor Bloch equations. Let's note, in passing that, contrary to the remark advanced by the authors in that reference, the virial theorem holds also for Coulomb-interacting particles even in the presence of the confining potential provided that the generalized form of the theorem is used (the virial "coefficient" is then not constant and depends on the form of the potential). Fig. 4a shows the dependence of $E_{b}$ on the size of the wire for balanced T-wires $(L=d)$ based on $\mathrm{Ga}_{0.6} \mathrm{Al}_{0.4} \mathrm{As}$ structure. Comparison of our results with those reported in that reference shows very good agreement. In Fig. 4b, we report the variations of the extensions of the exciton along the three characteristic directions versus the dimension of the wire for the same structure. This result shows that the extension along the stem direction is more sensitive to the confinement than extensions along the remaining axis while the stretching of the exciton is more marked along the arm direction.

The above findings show that the influence of the cross-section geometry of the wire is not minor. They
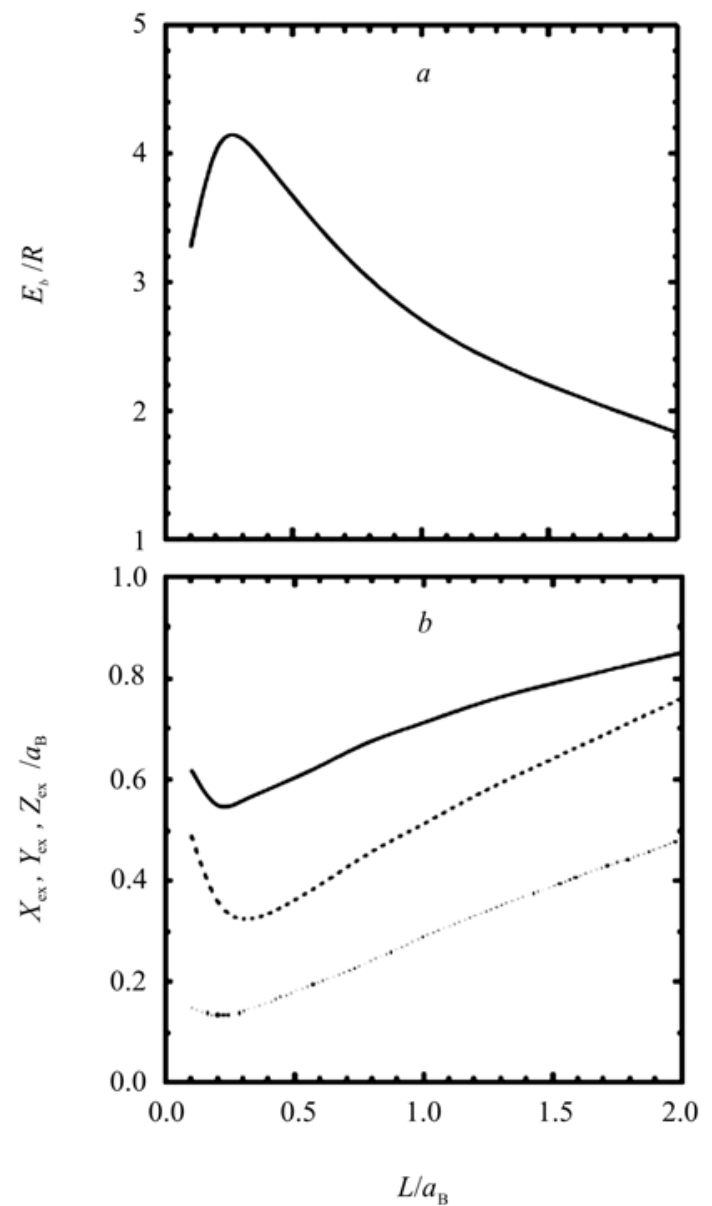

Fig. 4. $L$-dependence of the binding energy (a), $z$-extension, $x$ extension and $y$-extension (b) of the $X_{1 s}$ exciton for the TQWR based on $\mathrm{Al}_{0.4} \mathrm{Ga}_{0.6} \mathrm{As} / \mathrm{GaAs} / \mathrm{Al}_{0.4} \mathrm{Ga}_{0.6} \mathrm{As}$ structure. Inset the schematic diagram of the studied wire.

indicate that the square-like geometry may be advantageous for obtaining higher binding energies and more localized excitons in comparison with T-like geometry: enhancement rate of the binding energy may exceed $25 \%$ and stretching of the spatial extensions along the three directions are more pronounced. In Table 3, we have assembled, for comparison, some characteristics of the excitons in the two geometries: Tshaped and squared cross-section wires.

\subsection{Action of electric and magnetic fields}

As an original application of the method, we have examined the effects of a magnetic field, applied parallel

Table 3. Comparison of the exciton properties for SQWR and TQWR for $\mathrm{Ga}_{0.6} \mathrm{Al}_{0.4} \mathrm{As}$ structure. Between brackets indicated are the values of the well width associated with the extrema of the energy and $X, Y, Z$. The values are in the Rydberg energy and the Bohr radius of the bulk GaAs material (see Table 1).

\begin{tabular}{|c|c|c|c|c|}
\hline & $E_{b}^{\max }\left(L_{\max }\right)$ & $X_{\min }\left(L_{\min }\right)$ & $Y_{\min }\left(L_{\min }\right)$ & $Z_{\min }\left(L_{\min }\right)$ \\
\hline TQWR & $4.2(0.21)$ & $0.33(0.3)$ & $0.13(0.22)$ & $0.53(0.22)$ \\
\hline SQWR & $5.2(0.22)$ & $0.18(0.22)$ & $0.18(0.22)$ & $0.47(0.22)$ \\
\hline
\end{tabular}


to the $z$-axis and an electric field, applied parallel to the $x$-axis for SQWR. The effect of a constant electric field on the optical properties of quantum wells has been early investigated and it is known as Confinement Quantum Stark Effect. It results generally in a red shift of the PL peak positions with increasing electric field strength $F$ causing then a broadening of the exciton and decrease of its energy. This effect predominates especially for sufficiently wide wells where the confinement becomes weak [35]. These features are generally interpreted as a competition between the field-induced spatial separation between electron and hole along the direction of the field and the barrier-induced confinement. In this section, we will show how these properties are modified in the case of SQWRs where an additive confinement along the second growth-direction is present. The resolution of the problem is made in the framework of the model developed in Section 2 by calculating the $P$-function in terms of the one-particle wavefunctions where the field effect is included. On the other hand, it is now established that when a magnetic field is applied along the free-movement direction, the exciton in a quantum well squeezes, and its ground state energy shifts almost linearly with the field, as a consequence of the Landau quantization of the carrier states [36]. For T-shaped wires, analysis of magnetophotoluminescence measurements has concluded to a weak magnetic field-induced energy shift [37]. It has been demonstrated [38] that this small field-induced energy shifts is consistent with the hypothesis of weak T-junction confinement rather than that of $1 \mathrm{D}$ quantum confinement. We give in what follows the magnetic field-induced energy shifts of the exciton for SQWR and confront our results with those of T-wires. The calculation is performed, as in the case of the electric field effect, by determining the $P$-function in terms of the electron and hole states in the presence of the field. First, we derive the general equations when the electric and the magnetic fields are applied simultaneously. It is clear that the for configuration of the fields considered here, the movement of the free carriers is not affected by the fields along the $z$-axis. The fundamental one-particle wavefunctions can thus be chosen as $x$ - and $y$-dependent only. They were calculated variationally by using the following commonly used trial functions:

$\psi_{i}(F, B)=\psi_{i}^{0}\left(x_{i}, y_{i}\right)\left(1+\alpha_{i} x_{i}\right) \exp \left[-\beta_{i}\left(x_{i}^{2}+y_{i}^{2}\right)\right]$

where $\alpha_{i}$ and $\beta_{i}$ are the variational parameters and $\psi_{i}^{0}$ is the known wavefunction of the particle $i(i=e, h)$ in the absence of the field. The additive term in the Hamiltonian of the one-particle problem when the fields are applied may be written as follows:

$H_{i}^{F, B}=\frac{\gamma^{2}}{4 m_{i}}\left(x_{i}^{2}+y_{i}^{2}\right) \pm \eta x_{i}$

where “+” and "-_" signs hold for the electron and the hole respectively and the dimensionless parameters $\gamma$ and $\eta$ given by: $\gamma=\frac{e \hbar B}{2 \mu^{*} R}$ and $\eta=\frac{e a_{B} F}{R}$. The

calculation was performed for the case of the square-like wire based on $\mathrm{Al}_{0.4} \mathrm{Ga}_{0.6} \mathrm{As}$ system. The results are plotted in Fig. 5 for the binding energy and Fig. 6 for the spatial extensions of the exciton. Effects of electric (dotted line) and magnetic (dashed line) fields are reported in comparison with the unperturbed system (solid line). Following features can be noted:

(i) The influence of the electric field is effective only when the wire-width values exceed the limit of $0.5 a_{B}$. This limiting value is consistent with that which one can estimate after the pondering condition between the electric field and the confinement effects, i.e., $F e L>E_{i}(0)$ where $E_{i}(0)$ is the energy of the carrier in the absence of the field. When this condition is satisfied, the induced-electric field effect results in decreasing energy with higher rate than without field in addition of a broadening of the exciton even in the directions perpendicular to the field with larger relative expansion along the direction of the field.

(ii) The magnetic field remains ineffective as long as the well width values do not exceed the bulk-exciton Bohr radius $a_{B}$. For $L>a_{B}$, the magnetic field acts as an additive confinement by enhancing the binding energy and squeezing the exciton in the transversal plane as well as along the $z$-axis. These trends are compatible with those reported by A. Balandin et al. [39] where the exciton trial wave function was of the Gaussian type.

It is interesting to compare these results to those obtained by using the same method, when the exciton is confined in one direction only, i.e. in one quantum well. We have made this comparison for $\mathrm{Ga}_{0.6} \mathrm{Al}_{0.4} \mathrm{As}$ structure. The result was as follows. First, we have noted that the binding energy, as a function of the well-width, shows similar trends in the two configurations both when electric and magnetic fields are applied. More precisely, the relative decrease in energy (calculated for $L=200 \AA$ as illustration) for SQWR when the electric field $(F=50 \mathrm{kV} / \mathrm{cm})$ is applied is approximately twice

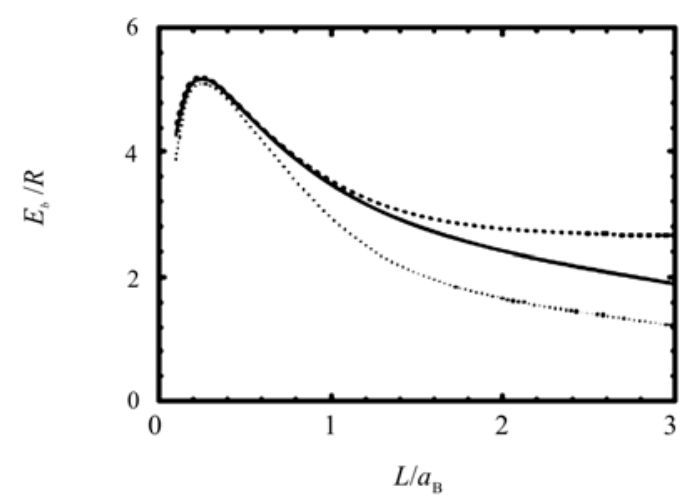

Fig. 5. $L$-dependence of the binding energy of the exciton $X_{1}$ confined in $\mathrm{Al}_{0.4} \mathrm{Ga}_{0.6} \mathrm{As} / \mathrm{GaAs} / \mathrm{Al}_{0.4} \mathrm{Ga}_{0.6}$ As squared wire in the presence of the electric field $F=50 \mathrm{kV} / \mathrm{cm}$ (dotted), the magnetic field $B=20 \mathrm{~T}$ (dashed) and without field (solid line). 


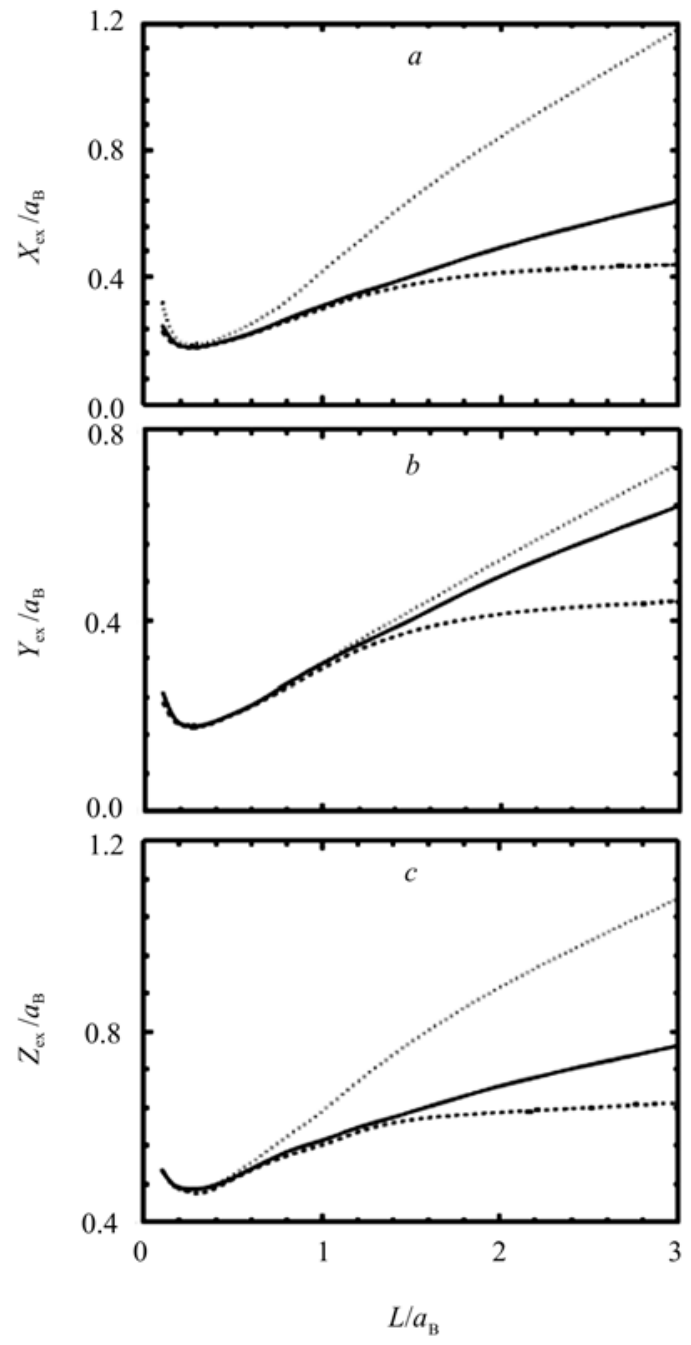

Fig. 6. $x$ - (a), $y$ - (b), and $z$ - (c) extensions of the exciton as functions of the wire size for the exciton confined in $\mathrm{Al}_{0.4} \mathrm{Ga}_{0.6} \mathrm{As} / \mathrm{GaAs} / \mathrm{Al}_{0.4} \mathrm{Ga}_{0.6} \mathrm{As}$ squared wire in the presence of the electric field $F=50 \mathrm{kV} / \mathrm{cm}$ (dotted), the magnetic field $B=20 \mathrm{~T}$ (dashed) and without field (solid line).

the same decrease calculated for the corresponding QW, while the relative expansion along the direction of the field falls of half passing from $100 \%$ in the QW to $60 \%$ in the SQWR. It shows that the induced-electric field effect in SQWRs is roughly twice marked than in corresponding QWs. On the other hand, the enhancement of the energy induced by the magnetic field is much more marked in the QW than in the SQWR where it can be neglected in the usual confinement regime. Similar behaviour was reported for T-shaped wires when compared with the corresponding quantum wells [38].

\section{Conclusions}

Using a variational calculation in the effective mass approximation and the two-band model, we have established a set of parametrized equations to be used for calculating exciton properties in quantum wires for any potential profile. Illustration is given for T-shaped and
SQWRs based on GaAlAs/GaAs structures. Comparison of the two systems shows that the confinement effects are slightly enforced for the squared geometry: the binding energies are higher and the spatial extensions more reduced except for the stem direction where the extension is lower in T-wire than in the corresponding squared wire. A new theoretical configuration where the confining potential is parabolic along the $x$-direction and rectangular with infinite height barrier along the $y$-direction was also considered. The study reveals that this confining potential configuration enhances the binding energy and enforces the localisation of the exciton with respect to the corresponding squared wire. The method was extended to study the effects of both electric and magnetic fields on the excitons in the SQWRs studied structures. It has been found that the induced-electric field effect results in decreasing energy with higher rate than without field in addition of a broadening of the exciton even in the directions perpendicular to the field with larger relative expansion along the direction of the field. It has been found also that the magnetic field acts as an additive confinement by enhancing the binding energy and squeezing the exciton in the transversal plane as well as along the $z$-axis. Comparison of these results with those obtained for corresponding $\mathrm{T}$-wires or quantum wires shows that the induced-electric field effect in SQWRs is roughly twice marked than in corresponding QWs while the enhancement of the energy induced by the magnetic field is much more marked in the QW than in the corresponding SQWR as well as in the corresponding T-wires.

\section{References}

1. S.S. Mao // Intern. J. Nanotechnology, 1, Nos. 1/2 (2004).

2. Biswajit Das, Pavan Singaraju // Infrared Phys. and Technol. 46(3), p. 209 (2005).

3. H. Akiyama // J. Phys.: Condens. Matter 10, p. 3095 (1998).

4. R. Schuster, H. Hajak, M. Reinwald, et al. // Phys. Stat. Sol. (c), 1(8), p. 2028 (2004).

5. H. Sakaki, Kato, H. Yoshimura // Appl. Phys. Lett. 57, p. 2800 (1990).

6. G.W. Bryant, P.S. Julienne and Y.B. Band // Superlattices and Microstructures, 20 (4), p. 601 (1996).

7. W. Langbein, H. Gislason and J.M. Hvam // Phys. Rev. B 54, p. 14595 (1996).

8. A.V. Kavokin // Semicond. Sci. Technol. 10, p. 606 (1995).

9. E.P. Pokatilov, V.A. Fonoberov, S.N. Balaban and V. M. Fomin // J. Phys.: Condens. Matter 12, p. 9037 (2000).

10. A. Zounoubi, K. EL Messaoudi, I. Zorkani and A. Jorio // Superlattices and Microstructures 30(4), p. 189 (2001).

11. J.W. Brown and H.N. Spector // Phys. Rev. B 35, p. 3009 (1987).

12. K. Chang and J.B. Xia // Phys. Rev. B 58, p. 2031, 1998. 
13. M.H. Degani and O. Hipolito // Phys. Rev. B 35, p. 9345 (1987).

14. Z.Y. Deng, T.R. Lai, J.K. Guo, H. Sun and S.W. Gu // J. Phys.: Condens. Matter 6, p. 5681 (1994).

15. K. Siantidis, V.M. Axt, J. Wühr and T. Kuhn // Phys. Stat. Sol. (a) 190(3), p. 743 (2002).

16. Z.Y. Deng, H. Sun and S.W. Gu // J. Phys.: Condens. Matter, 4, p. 6549 (1992).

17. L.C. Lew, Yan Voon and M. Willatzen // Semicond. Sci. Technol. 10, p. 416 (1995).

18. F.A.P. Osorio, M.H. Degani and O. Hipolito // Phys. Rev. B 37(3), p. 1402 (1988).

19. M. de Dios-Leyva and L.E. Oliveira // J. Phys.: Condens. Matter 13, p. 9471 (2001).

20. K. Kim and B. Lee // J. Opt. Soc. Amer. B. 19(5), p. 1039 (2002).

21. F.V. Kyrychenko and J. Kossut // Phys. stat. sol. (b) 229(1), p. 557 (2002).

22. L. Chen, H. Falk, P.J. Klar, et al. // Phys. stat. sol. (b) 229(1), p. 31 (2002).

23. K. Komori, G.R. Hayes, B. Deveaud, X.L. Wang, M. Ogura and M. Watanabe // Phys. stat. sol. (a) 190(3), p. 855 (2002).

24. L. Bányai, I. Galbraith, C. Ell and H. Hang // Phys. Rev. B 36(11), p. 6099 (1987).

25. G. Goldoni, F. Rossi, and E. Molinari // Phys. stat. sol. (b) 164, p. 265 (1997).
26. T. Sogawa, H. Ando, S. Ando, and H. Kanbe et al. // Phys. Rev. B 56, p. 1958 (1997).

27. J. Diouri, A. Taqi, A. El Haddad, M. Katih and E. Feddi // Semicond. Sci. Technol. 18, p. 377 (2003).

28. M. Tsetseri and G.P. Triberis // Superlatt. and Microstruct. 32, p. 79 (2002).

29. G.W. Bryant // Phys. Rev. B, 37(15), p. 8763 (1988).

30. A.N. Gordeyev and S.C. Chhjlany // J. Phys. A 30, p. 6893 (1997).

31. M. Combescot and T. Guillet // Eur. Phys. J. B 34, p. 9 (2003).

32. G. Goldoni, F. Rossi, E. Molinari, A. Fasolino, R. Rinaldi and R. Cingolani // Appl. Phys. Lett. 69, p. 2965 (1996).

33. F. Vouilloz, D.Y. Obesli, M.A. Dupertuis, A. Gustafsson, F. Reinhardt and E. Kapon // Phys. Rev. B 57, p. 12378 (1998).

34. T. Someya, H. Akiyama, and H. Sakaki // Phys. Rev. Lett. 76(16), p. 2965 (1996).

35. D-S. Chuu and Y-T. Shih // Phys. Rev. B 44, p. 8054 (1991).

36. W. Edelstein // Phys. Rev. B, 39, p. 7697 (1989).

37. T. Someya, H. Akiyama and H. Sakaki // Solid State Commun. 108, p. 923 (1998).

38. G.W. Bryant and Y.B. Band // Phys. Rev. B 63, p. 115304 (2001).

39. A. Balandin and Bandyopadhyay // Phys. Rev. B 52, p. 8312 (1995). 